\title{
Clinical value of pathologic examination of non-neoplastic kidney in patients with upper urinary tract malignancies
}

Jee Wan Wee ${ }^{1}$, Hye Ran Kang ${ }^{1}$, Soon Hyo Kwon ${ }^{1}$, Jin Seok Jeon ${ }^{1}$, Dong Cheol Han ${ }^{1}$, So-Young Jin ${ }^{2}$, Won Jae Yang ${ }^{3}$, and Hyunjin Noh $^{1}$

Departments of ${ }^{1}$ Internal Medicine, ${ }^{2}$ Pathology, and ${ }^{3}$ Urology, Soon Chun Hyang University College of Medicine, Seoul, Korea

Received: December 8, 2015

Revised : January 4, 2016

Accepted: January 5, 2016

\section{Correspondence to}

Hyunjin Noh, M.D.

Department of Internal

Medicine, Soon Chun Hyang

University College of Medicine, 59

Daesagwan-ro, Yongsan-gu, Seoul 04401, Korea

Tel: +82-2-709-9177

Fax: +82-2-792-5812

E-mail: nohneph@schmc.ac.kr
Background/Aims: While surgical resection remains the standard of care in the treatment of upper urinary tract malignancies, nephrectomy is a risk factor for the development of chronic kidney disease (CKD). The aim of this study was to determine whether histologic evaluation of non-neoplastic kidney could enable early identification of unrecognized kidney disease and could be of prognostic value in predicting postoperative renal outcomes.

Methods: We retrospectively analyzed 51 patients with upper urinary tract malignancies who received uninephrectomy or uninephroureterectomy. A thorough pathologic evaluation of non-neoplastic kidney including special stains, immunofluorescence, and electron microscopic studies was performed. The degree of parenchymal changes was graded from o to 15 .

Results: Of 51 patients, only 13 showed normal kidney pathology. Fifteen patients showed glomerular abnormalities, 14 showed diabetic nephropathy, and 11 showed vascular nephropathy. There was one case each of reflux nephropathy and chronic pyelonephritis. The median histologic score was 5 points. Only $25.4 \%$ of patients had $\leq 3$ points. Score more than 5 was observed in $47.1 \%$ of patients. Postoperative estimated glomerular filtration rate (eGFR) at 3 to 36 months were obtained from $90.2 \%$ of patients, and of those, $34.8 \%$ had de novo CKD. Since no one had CKD in partial nephrectomized patients, we determined risk factors for CKD in radical nephrectomized patients. Cox regression analysis revealed that postoperative AKI, preoperative eGFR, and histologic score of non-neoplastic kidney were the independent predictors for CKD.

Conclusions: We conclude that routine pathologic evaluation of non-neoplastic kidney provides valuable diagnostic and prognostic information.

Keywords: Kidney neoplasms; Nephrectomy; Non-neoplastic pathology; Renal insufficiency, chronic

\section{INTRODUCTION}

The incidence of kidney cancer has been dramatically increasing worldwide during the past decade. Renal cell carcinoma (RCC) accounts for $90 \%$ of kidney cancer and approximately $3 \%$ of all adult malignancies in the United States [1]. The Korea Central Cancer Registry reported that 3,989 patients were newly registered for RCC in 2011, accounting for $1.8 \%$ of all adult malignancies [2]. Due to recent advances in diagnostic imaging techniques, early 
detection of localized RCC has been increased and now comprises more than half of newly diagnosed RCCs [3]. Since oncologic outcomes are excellent for early stage tumors when treated with a nephrectomy, there has been an emerging interest in postoperative renal outcomes [4]. The risks of new onset chronic kidney disease (CKD) or accelerated CKD after radical nephrectomy have been shown to be as high as $65 \%$ [5]. In this regards, nephron-sparing surgeries have been proposed as the standard of care for small renal cortical tumors $[6,7]$.

$\mathrm{CKD}$ is a public health problem and its prevalence is steadily rising [8]. CKD is associated with increased cardiovascular complications, lower survival, and a reduced quality of life $[9,10]$. The Third National Health and Nutrition Examination Survey reported the prevalence of CKD in the United States adult population to be $11 \%$ (19.2 million) [11]. It is well known that the cost of health services for patients with CKD is much higher than that for patients without CKD [12]. Therefore, early recognition of the patients at risk for CKD and prompt institution of treatment may be of considerable importance.

Given the fact that RCC and CKD share the risk factors such as hypertension (HTN), diabetes mellitus (DM), smoking, and advanced age [13], postoperative decline in renal functions might be more pronounced if unidentified intrinsic renal abnormalities were present at the time of nephrectomy. Furthermore, a solitary kidney is considered as a contraindication to percutaneous biopsy and the safety of this procedure in patients with partial nephrectomy has not been demonstrated [14]. Therefore, with thorough examination of non-neoplastic renal parenchyma, one can recognize the patients at risk for CKD and predict their clinical courses.

The aim of this study was to determine whether histologic evaluation of non-neoplastic kidney could enable early identification of unrecognized kidney disease and could be of prognostic value in predicting postoperative renal outcomes.

\section{METHODS}

\section{Participants and measurements}

We studied a cohort of 49 patients with RCC and seven patients with urothelial cancer received uninephrectomy or uninephroureterectomy between January 2010 and January 2014 at Soon Chun Hyang University Hospital, Seoul, Korea. All nephrectomized patients due to upper urinary tract malignancies were included, with the exception of patients with end stage renal disease (ESRD, $n=4)$ and those with a transplanted kidney with RCC in the native kidney $(n=1)$. The study protocol was approved by the Institutional Review Board of the Soon Chun Hyang University Hospital.

\section{Covariates and definitions}

Baseline variables including demographic features (age and sex), medical history (DM, HTN, cardiovascular disease [CVD], and previous malignancies), smoking status, body mass index (BMI), and laboratory data (serum creatinine $[\mathrm{sCr}]$, proteinuria, hematuria, hepatitis $\mathrm{B}$ surface antigen, and hepatitis $\mathrm{C}$ antibody) were examined. Smoking status and medical history were extracted from electronic medical record database. History of DM and HTN was defined by clinical diagnosis. CVD was defined as the clinical diagnosis of heart failure, acute or chronic ischemic heart disease, and cerebrovascular disease. BMI was calculated by the baseline enrolled measurement using the formula weight in kilograms divided by the square of their height in meters. The definitions of CKD and acute kidney injury (AKI) were based on the Kidney Disease Improving Global Outcomes guidelines. CKD was defined as abnormalities of kidney function (estimated glomerular filtration rate $[\mathrm{eGFR}]<60 \mathrm{~mL} /$ $\min / 1.73 \mathrm{~m}^{2}$ ) over 3 months, and AKI was defined as an increase in $\mathrm{sCr}$ by $50 \%$ within 7 days, or an increase in sCr by $0.3 \mathrm{mg} / \mathrm{dL}$ within 2 days. The eGFR was calculated using the Chronic Kidney Disease Epidemiology Collaboration creatinine equation [15], as follows: $141 \times$ $\min (\mathrm{sCr} / \kappa, 1)^{\alpha} \times \max (\mathrm{sCr} / \kappa, 1)^{-1.209} \times 0.993^{\text {Age }}[\times 1.018$ if female] [ $\times 1.159$ if black], where $\mathrm{sCr}$ is in $\mathrm{mg} / \mathrm{dL}, \kappa$ is 0.7 for females and 0.9 for males, $\alpha$ is -0.329 for females and -0.411 for males, $\min$ is the minimum of $\mathrm{sCr} / \kappa$ or 1 , and max is the maximum of $\mathrm{s} \mathrm{Cr} / \mathrm{\kappa}$ or 1 . Proteinuria or hematuria was defined as detection by dipstick.

\section{Pathologic review}

The routine pathologic evaluation of non-neoplastic tissues was performed. After nephrectomy, fractions of tumor and non-neoplastic cortical tissue samples from surgical specimens were collected. Representative non-neoplastic sections were examined at a significant distance from the 
tumor (at a minimum $2 \mathrm{~cm}$ away). Under light microscopy, slides were reviewed following staining with hematoxylin and eosin, as well as periodic acid-Schiff, Masson's trichrome, and Jones methenamine silver. Immunofluorescent (IF) microscopy was performed on frozen tissue sections. Immunoglobulin G (IgG), IgA, IgM, complement component $\mathrm{C}_{3}$, and $\mathrm{C}_{1} \mathrm{q}$ were stained as usual. Routine electron microscopy (EM) was performed using 1-mm sections excised from fresh tissue, which was fixed with $2.5 \%$ glutaraldehyde solution. Pathologic review was performed by a renal pathologist (SYJ). We diagnosed hypertensive nephrosclerosis which has globally sclerotic glomeruli and patchy atrophic tubules of dependent nephron, surrounded by marked interstitial fibrosis and chronic inflammation with moderate to severe arteriosclerosis or arteriolosclerosis. Ischemic nephropathy was defined as lesions secondary to renal artery stenosis, tubular atrophy of distinctive "endocrinization" pattern affecting proximal tubules, accompanied by mild to moderate interstitial fibrosis, resulting in crowded glomeruli showing relative dilation of Bowman's space, variable degree of collapse of capillary loops, and wrinkling of capillary wall.

A complete histologic examination included an evaluation of the presence of glomerulosclerosis, interstitial fibrosis, tubular atrophy, and vascular pathology. All samples were graded according to a semi-quantitative scoring system [16]. This semi-quantitative scoring system ranged from a minimum of o to a maximum of 15 points.

\section{Statistical analysis}

All statistical analyses were performed using SPSS version 18.o (SPSS Inc., Chicago, IL, USA). Variables are presented as the medians with interquartile range or mean \pm standard deviation. Fisher exact test and chi-square test were used for categorical covariates, and Student $t$ test was used for continuous covariates. We also used logistic regression analyses to investigate independent risk factors for the occurrence of postoperative AKI. Multivariable Cox proportional hazards regression was used to determine the association of clinical characteristics and pathologic findings of non-neoplastic kidney with the development or acceleration of CKD. All $p$ values less than 0.05 were considered to indicate statistical significance.

\section{RESULTS}

\section{Baseline characteristics}

Patient characteristics at the time of surgery are listed in Table 1. The median patient age was 63 years (53 to 73) and the median BMI was $24.3 \mathrm{~kg} / \mathrm{m}^{2}$ (22.7 to 26.6). Twenty-six patients $(51 \%)$ had HTN, 20 patients $(39.2 \%)$ had DM, and nine patients (17.6\%) had CVD. There were 13 patients $(25.5 \%)$ with a history of malignancy. Of 51 patients, 22 (43.1\%) were smokers or ex-smokers. Five patients (9.8\%) had been diagnosed with hepatitis B and one patient ( $2 \%)$ with hepatitis C. Thirty-two patients were treated with radical nephrectomy, 12 patients with partial nephrectomy, and seven patients with nephroureterectomy. Initial urinalysis was performed in $50 \mathrm{pa}-$ tients (98\%). Of those, 12 (23.5\%) had proteinuria and 18 (35.3\%) had hematuria. The median preoperative sCr was $0.81 \mathrm{mg} / \mathrm{dL}$ (0.71 to 1.01) and preoperative eGFR was 92.1 $\mathrm{mL} / \mathrm{min} / 1.73 \mathrm{~m}^{2}$ (72.8 to 104.8 ).

\section{Non-neoplastic pathologic findings}

Pathologic reports of non-neoplastic kidney were obtained from 44 RCC patients treated with nephrectomy and seven urothelial cancer patients treated with nephro-

Table 1. Baseline patient characteristics at the time of nephrectomy $(n=51)$

\begin{tabular}{lc}
\hline Characteristic & Value \\
\hline Age, yr & $63(53-73)$ \\
Male sex & $38(74.5)$ \\
Body mass index, $\mathrm{kg} / \mathrm{m}^{2}$ & $24.3(22.7-26.6)$ \\
Hypertension & $26(51)$ \\
\hline Diabetes mellitus & $20(39.2)$ \\
Cardiovascular disease & $9(17.6)$ \\
Previous malignancy & $13(25.5)$ \\
Smoking history & $22(43.1)$ \\
Hepatitis B & $5(9.8)$ \\
Hepatitis C & $1(2)$ \\
Preoperative proteinuria & $12(23.5)$ \\
Preoperative hematuria & $18(35.3)$ \\
\hline Preoperative creatinine, $\mathrm{mg} / \mathrm{dL}$ & $0.81(0.71-1.01)$ \\
\hline Preoperative eGFR, mL/min/1.73 $\mathrm{m}^{2}$ & $92.1(72.8-104.8)$ \\
\hline
\end{tabular}

Values are presented as median (interquartile range) or number (\%).

eGFR, estimated glomerular filtration rate. 
ureterectomy. Of 51 patients, only 13 (25.5\%) showed normal kidney pathology. Forty-two cases of patholog-

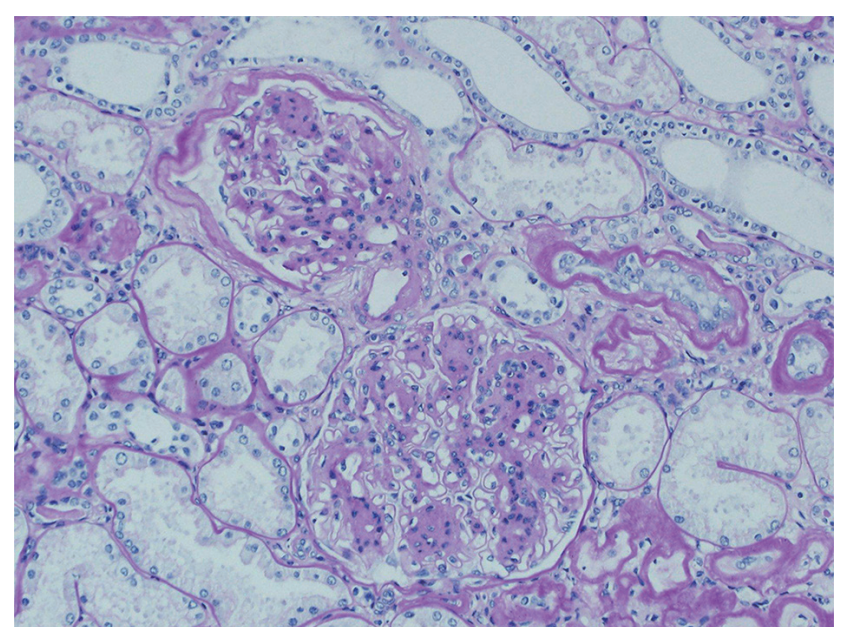

Figure 1. Nodular glomerulosclerosis and hyaline arteriolosclerosis in a case of diabetic nephropathy (periodic acid-Schiff, $\times 200$ ). ic diagnoses were obtained in 38 patients because four patients had dual diagnoses. Fifteen patients (29.4\%) showed glomerular abnormality, 14 (27.5\%) showed diabetic nephropathy (Fig. 1), and 11 showed (21.6\%) vascular nephropathy. In glomerular abnormalities, there were nine cases (17.6\%) of IgA nephropathy, five cases (9.8\%) of Ciq deposit (Fig. 2), and one case (2\%) of membranous glomerulonephritis (Fig. 3). Vascular nephropathy included eight cases (15.7\%) of hypertensive nephrosclerosis and three cases $(5.9 \%)$ of ischemic nephropathy. There was one case each of reflux nephropathy and chronic pyelonephritis (2\%) (Table 2).

Semi-quantitative evaluation of non-neoplastic kidney is shown in Table 3. Glomerulosclerosis was present in $94.1 \%$ of all patients, predominantly of grade 1 (56.9\%). Severe glomerulosclerosis (> 20\%) was present in $13.7 \%$ of cases. Interstitial fibrosis was present in $51 \%$, predominantly of grade 1 (39.2\%). Fifty-one percent of patients showed tubular atrophy and grade 1 was pre-
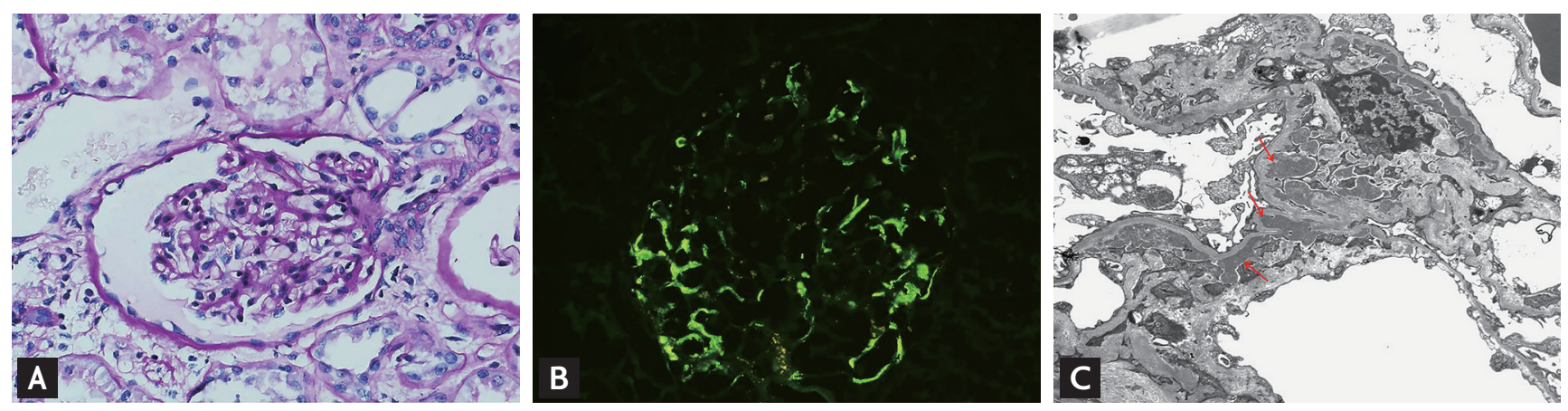

Figure 2. A case of Ciq nephropathy. (A) Nearly normal appearing glomerulus with mesangial prominence (periodic acid-Schiff, ×400). (B) Positive immunofluorescence staining for Ciq (×400). (C) Multiple mesangial electron dense deposits (arrows) on electron microscopy $(\times 2,500)$.
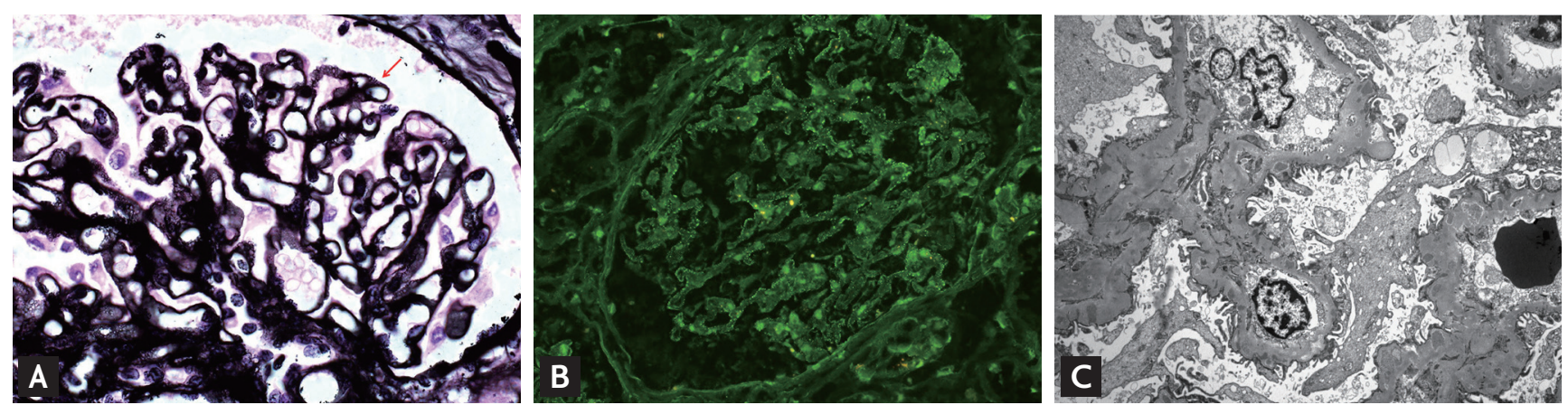

Figure 3. (A) Few subepithelial spikes (arrow) and multiple intramembranous holes in a case of membranous glomerulonephritis (methenamine silver stain, $\times 600$ ). (B) Weak granular staining for immunoglobulin $\mathrm{G}$ along the peripheral capillary wall (immunofluorescence, $\times 400$ ). (C) Multiple small subepithelial and intramembranous electron dense deposits on electron microscopy $(\times 3,000)$. 
Table 2. Pathologic review of non-neoplastic kidney in patients with nephrectomy

\begin{tabular}{lc}
\hline Pathologic diagnosis & No. of cases ${ }^{\mathrm{a}}$ \\
\hline Normal & $13(25.5)$ \\
Glomerular abnormality & $15(29.4)$ \\
\hline Immunoglobulin A nephropathy & $9(17.6)$ \\
C1q deposit & $5(9.8)$ \\
Membranous glomerulonephritis & $1(2.0)$ \\
Diabetic nephropathy & $14(27.5)$ \\
Vascular nephropathy & $11(21.6)$ \\
\hline Hypertensive nephrosclerosis & $8(15.7)$ \\
Ischemic nephropathy & $3(5.9)$ \\
Reflux nephropathy & $1(2.0)$ \\
Chronic pyelonephritis & $1(2.0)$ \\
\hline
\end{tabular}

Values are presented as number (\%).

${ }^{a}$ The total number of findings exceed 100\% because four patients had dual diagnoses. dominant (39.2\%). Of note, fibrous intimal thickening was observed in most of the cases (94.1\%), predominantly of grade $2(49 \%)$. Hyaline arteriolar thickening was present in $78.4 \%$. Grade 1 was predominant (49\%) and grade 2 was the second (21.6\%), followed by grade $3(7.8 \%)$. After scoring each of the five components, we calculated the total score. The median total score was 5 points ( 3 to 7 ). Only $25.4 \%$ of patients had $\leq 3$ points. Score more than 5 points was observed in $47.1 \%$ of patients.

\section{Postoperative renal function outcome}

Twenty-eight patients (54.9\%) experienced postoperative AKI. Among these patients, $15(53.6 \%)$ had HTN and 13 (46.4\%) had DM. Five patients (17.9\%) had a history of CVD. There was no correlation between the occurrence of postoperative AKI and clinical variables except older age $(p=0.026)$, male gender $(p=0.043)$, and radical

Table 3. Semi-quantitative evaluation of non-neoplastic kidney

\begin{tabular}{|c|c|}
\hline Histologic scoring & All patients, \% \\
\hline \multicolumn{2}{|l|}{ Glomerular global sclerosis } \\
\hline o: none globally sclerosed & 5.9 \\
\hline 1: 1\%-10\% global glomerulosclerosis & 56.9 \\
\hline 2: $11 \%-20 \%$ global glomerulosclerosis & 23.5 \\
\hline $3:>20 \%$ global glomerulosclerosis & 13.7 \\
\hline \multicolumn{2}{|l|}{ Interstitial fibrosis } \\
\hline $0:<5 \%$ of renal tissue replaced by fibrous connective tissue absent & 49 \\
\hline 1: $6 \%-25 \%$ of renal tissue replaced by fibrous connective tissue & 39.2 \\
\hline 2: $26 \%-50 \%$ of renal tissue replaced by fibrous connective tissue & 7.8 \\
\hline $3:>50 \%$ of renal tissue replaced by fibrous connective tissue & $3 \cdot 9$ \\
\hline \multicolumn{2}{|l|}{ Tubular atrophy } \\
\hline o: absent & 49 \\
\hline $1:<25 \%$ of tubule affected & 39.2 \\
\hline 2: $26 \%-50 \%$ of tubule affected & 7.8 \\
\hline $3:>50 \%$ of tubule affected & $3 \cdot 9$ \\
\hline \multicolumn{2}{|l|}{ Fibrous intimal thickening } \\
\hline o: absence of chronic vascular changes & $5 \cdot 9$ \\
\hline $1:<25 \%$ narrowing of the vascular luminal area by fibrointimal thickening & 39.2 \\
\hline 2: similar changes with narrowing of the vascular luminal area from $26 \%$ to $50 \%$ & 49 \\
\hline 3: similar changes with more than $50 \%$ narrowing of the vascular luminal area & $5 \cdot 9$ \\
\hline \multicolumn{2}{|l|}{ Hyaline arteriolar thickening } \\
\hline o: absence & 21.6 \\
\hline $\begin{array}{l}\text { 1: replacement of degenerated smooth muscle cells by hyaline deposits present in onlyone arteriole, no } \\
\text { circumferential involvement }\end{array}$ & 49 \\
\hline 2: similar changes in more than one arteriole, but no circumferential involvement & 21.6 \\
\hline 3: similar changes with circumferential involvement, independent of the number of arterioles involved & 7.8 \\
\hline
\end{tabular}


Table 4. Univariate analysis for clinical variables affecting acute kidney injury

\begin{tabular}{lccc}
\hline \multirow{2}{*}{ Variable } & \multicolumn{2}{c}{ Acute kidney injury } & \multirow{2}{*}{ pvalue } \\
\cline { 2 - 3 } Age, yr & Yes $(\mathrm{n}=28)$ & No $(\mathrm{n}=23)$ & 0.026 \\
Male sex & $65.7 \pm 11.1$ & $58.8 \pm 10.1$ & 0.043 \\
Body mass index, $\mathrm{kg} / \mathrm{m}^{2}$ & $24(85.7)$ & $14(60.9)$ & 0.836 \\
Hypertension & $24.6 \pm 3.1$ & $24.4 \pm 3.5$ & 0.683 \\
Diabetes mellitus & $15(53.6)$ & $11.7(47.8)$ & 0.244 \\
Cardiovascular disease & $13(46.4)$ & $7(30.4)$ & $>0.999$ \\
Smoking & $5(17.9)$ & $4(17.4)$ & 0.964 \\
Preoperative proteinuria & $12(42.9)$ & $10(43.5)$ & 0.852 \\
Radical nephrectomy & $7(25.0)$ & $5(22.7)$ & 0.002 \\
Preoperative eGFR, $\mathrm{mL} / \mathrm{min} / 1.73 \mathrm{~m}^{2}$ & $26(92.9)$ & $13(56.5)$ & 0.116 \\
Pathologic abnormality & $86.0 \pm 20.0$ & $95.9 \pm 24.4$ & 0.090 \\
Histologic score & $23(82.1)$ & $14(60.9)$ & 0.287 \\
\hline
\end{tabular}

Values are presented as mean \pm SD or number (\%).

eGFR, estimated glomerular filtration rate.

Table 5. Multivariate logistic regression analysis for clinical variables affecting acute kidney injury

\begin{tabular}{lll}
\hline Variable & OR $(95 \%$ CI & $p$ value \\
\hline Age & $1.11(1.01-1.22)$ & 0.029 \\
Sex, male vs. female & $0.15(0.02-1.28)$ & 0.083 \\
Hypertension, yes vs. no & $0.27(0.04-1.99)$ & 0.199 \\
Diabetes mellitus, yes vs. no & $1.44(0.22-9.36)$ & 0.704 \\
Smoking, yes vs. no & $0.69(0.11-4.19)$ & 0.687 \\
Surgical method, radical vs. partial nephrectomy & $15.31(1.84-127.23)$ & 0.012 \\
Preoperative proteinuria, yes vs. no & $0.38(0.04-3.89)$ & 0.412 \\
Preoperative eGFR & $1.00(0.96-1.04)$ & 0.971 \\
Pathologic abnormality, yes vs. no & $1.86(0.27-12.57)$ & 0.527 \\
Histologic score & $1.06(0.76-1.46)$ & 0.735 \\
\hline
\end{tabular}

OR, odds ratio; CI, confidence interval; eGFR, estimated glomerular filtration rate.

nephrectomy $(p=0.002)$ compared to partial nephrectomy (Table 4). Logistic regression analysis was conducted with variables of age, gender, HTN, DM, smoking, surgical method, preoperative proteinuria, preoperative eGFR, presence of pathologic diagnosis of non-neoplastic tissue, and semi-quantitative histologic score (Table 5). It revealed that older age $(p=0.029)$ and radical nephrectomy $(p=0.012)$ were the independent risk factors for postoperative AKI. Data on eGFR at 3 to 36 months after surgery were obtained from 46 patients (90.2\%); 16 patients (34.8\%) had newly developed CKD stage 3 to 5 $\left(\right.$ eGFR $\left.<60 \mathrm{~mL} / \mathrm{min} / 1.73 \mathrm{~m}^{2}\right)$ after surgery. Four patients initially had CKD stage 3 at the time of nephrectomy. In three of four patients with pre-existing CKD, renal function was deteriorated by 27.5, 21.7, and $6.4 \mathrm{~mL} / \mathrm{min} / 1.73$ $\mathrm{m}^{2}$ reduction of eGFR. Among the 19 patients who had de novo or accelerated CKD, 14 (73.7\%) experienced postoperative AKI. The prevalence of CKD was significantly higher in patients with postoperative AKI than those without AKI (58.3\% vs. $22.7 \%, p=0.019$ ), in patients with preoperative proteinuria than those without proteinuria (75\% vs. $30.3 \%, p=0.015)$, in patients who underwent radical nephrectomy versus partial nephrectomy (54.3\% vs. $\circ \%, p=0.001$ ), in patients with abnormal pathologic 
Table 6. Univariate analysis for clinical variables affecting chronic kidney disease

\begin{tabular}{lccc}
\hline \multirow{2}{*}{ Variable } & \multicolumn{2}{c}{ Chronic kidney disease } & \multirow{2}{*}{ No value } \\
\cline { 2 - 3 } Age, yr & Yes $\left(\mathrm{n}=19^{\mathrm{a}}\right)$ & $59)$ & 0.089 \\
Male sex & $65.2 \pm 12.0$ & $19(70.4)$ & 0.320 \\
Body mass index, $\mathrm{kg} / \mathrm{m}^{2}$ & $16(84.2)$ & $24.2 \pm 3.0$ & 0.897 \\
Hypertension & $24.3 \pm 3.4$ & $12(44.4)$ & 0.245 \\
Diabetes mellitus & $12(63.2)$ & $10(37)$ & 0.767 \\
Cardiovascular disease & $8(42.1)$ & $4(14.8)$ & 0.700 \\
Smoking & $4(21.1)$ & $12(44.4)$ & 0.756 \\
Preoperative proteinuria & $10(52.6)$ & $3(11.5)$ & 0.015 \\
Radical nephrectomy & $9(47.4)$ & $16(59.3)$ & 0.001 \\
Postoperative AKI & $19(100.0)$ & $10(37)$ & 0.019 \\
Preoperative eGFR, mL/min $/ 1.73 \mathrm{~m}^{2}$ & $14(73.7)$ & $99.3 \pm 21.6$ & 0.002 \\
Pathologic abnormality & $78.2 \pm 19.8$ & $16(59.3)$ & 0.044 \\
\hline Histologic score & $17(89.5)$ & $4.0 \pm 2.0$ & 0.002 \\
\hline
\end{tabular}

Values are presented as mean \pm SD or number (\%).

AKI, acute kidney injury; eGFR, estimated glomerular filtration rate.

${ }^{a}$ Including 16 patients with de novo chronic kidney disease (CKD) and three patients with accelerated CKD.

diagnosis than those without diagnosis ( $51.5 \%$ vs. $15.4 \%$, $p=0.044$ ), and in patients who have lower preoperative eGFR $(p=0.002)$ and higher semi-quantitative histologic score $(p=0.002)$. Clinical characteristics between the patients with or without CKD are compared in Table 6. Since no one has developed CKD in partial nephrectomized patients, we determined independent risk factors for CKD in radical nephrectomized patients. Cox regression analysis with variables of age, HTN, DM, smoking status, preoperative proteinuria, postoperative AKI, preoperative eGFR, presence of pathologic diagnosis of non-neoplastic kidney, and semi-quantitative histologic score was done. In this study, preoperative eGFR (hazard ratio [HR], 0.95; 95\% confidence interval [CI], 0.92 to $0.98 ; p=0.004)$, postoperative AKI (HR, 7.03; 95\% CI, 2.01 to $24.61 ; p=0.002$ ), and semi-quantitative histologic score (HR, 1.23; 95\% CI, 1.00 to 1.50; $p=0.047$ ) were the independent predictors for CKD in radial nephrectomized patients. Characteristics of 19 patients who had de novo or accelerated CKD are shown in Table 7.

\section{DISCUSSION}

In this study, pathologic examination of non-neoplas- tic kidney revealed that $74.5 \%$ of patients underwent nephrectomy showed definitive morphological abnormalities. The most frequent pathologic finding was glomerular abnormality followed by diabetic and vascular nephropathies. Since almost $40 \%$ to $50 \%$ of our study population had HTN and DM and the median age was 63 years, diabetic nephropathy and vascular diseases would be expected to be present. However, the frequency of glomerular abnormalities was much higher than the previous studies. Two separate analyses demonstrated the presence of abnormal pathologic findings in $61.8 \%$ [17] and 15\% [18] of the kidneys where the non-neoplastic portions from tumor nephrectomy specimens were reviewed. In both studies, the most common findings were diabetic nephropathy and vascular-related changes. There were only two cases of IgA nephropathy out of 110 cases reviewed [17] and one case each of proliferative glomerulonephritis and membranous nephropathy out of 381 cases reviewed [18]. We believe that the higher diagnostic rate of glomerular abnormality in our study is related to our methodology. Unlike the previous reports [17-19] that performed special studies only in selected cases, all non-neoplastic tissue samples were routinely subjected to IF and EM, which detect unsuspected immune deposits, glomerular architecture changes, and 
Table 7. Characteristics of patients who had de novo or accelerated chronic kidney disease

\begin{tabular}{|c|c|c|c|c|c|c|c|}
\hline No. & $\begin{array}{c}\text { Sex/Age, } \\
\text { yr }\end{array}$ & $\begin{array}{l}\text { DM/ } \\
\text { HTN }\end{array}$ & $\begin{array}{l}\text { BMI, } \\
\mathrm{kg} / \mathrm{m}^{2}\end{array}$ & $\begin{array}{c}\text { Baseline Cr } \\
(\mathrm{mg} / \mathrm{dL}) / \mathrm{eGFR} \\
\left(\mathrm{mL} / \mathrm{min} / 1.73 \mathrm{~m}^{2}\right)\end{array}$ & $\begin{array}{c}\text { Final Cr } \\
(\mathrm{mg} / \mathrm{dL}) / \mathrm{eGFR} \\
\left(\mathrm{mL} / \mathrm{min} / 1.73 \mathrm{~m}^{2}\right)\end{array}$ & $\begin{array}{c}\text { Time to } \\
\text { development of } \\
\text { eGFR }<60 \mathrm{~mL} / \\
\min / 1.73 \mathrm{~m}^{2} \text {, day }\end{array}$ & Pathologic diagnosis \\
\hline 1 & $\mathrm{M} / 78$ & $+/+$ & 26.4 & $0.77 / 103.9$ & $1.79 / 35 \cdot 3$ & 1 & Normal \\
\hline 2 & $\mathrm{M} / 46$ & $-1-$ & 23.6 & $0.96 / 89.6$ & $1.59 / 50.9$ & 18 & Ciq deposit \\
\hline 3 & $\mathrm{M} / 59$ & $+/+$ & $24 \cdot 5$ & $1.55 / 49$ & $2.99 / 21.5$ & $\mathrm{O}^{\mathrm{a}}$ & Ciq deposit, DN \\
\hline 4 & $\mathrm{M} / 43$ & $-1-$ & 19.5 & $0.77 / 117.2$ & $2.57 / 29.1$ & 366 & $\mathrm{HN}$ \\
\hline 5 & $\mathrm{M} / 84$ & $+/-$ & $19 \cdot 3$ & $1.1 / 67.8$ & $3.22 / 19.6$ & 1 & IN \\
\hline 6 & $\mathrm{~F} / 66$ & $+/-$ & 26.6 & $0.7 / 89$ & $0.99 / 58.7$ & 549 & Ciq deposit \\
\hline 7 & $\mathrm{M} / 66$ & $-1-$ & $27 \cdot 9$ & $0.86 / 94.6$ & $1.28 / 57.1$ & 199 & Ciq deposit \\
\hline 8 & $\mathrm{~F} / 68$ & $+/+$ & 24.1 & $1.01 / 57.9$ & $1.46 / 36.2$ & $\mathrm{O}^{\mathrm{a}}$ & $\mathrm{DN}$ \\
\hline 9 & $\mathrm{~F} / 73$ & $+/+$ & 22.3 & $0.64 / 96.7$ & $0.98 / 59.1$ & 30 & $\mathrm{DN}$ \\
\hline 10 & $M / 64$ & $-1+$ & 22.0 & $1.0 / 80$ & $1.96 / 34.6$ & 1 & Reflux nephropathy \\
\hline 11 & $\mathrm{M} / 73$ & $+/+$ & $27 \cdot 3$ & $1.1 / 69.7$ & $2.36 / 26$ & 1 & IN \\
\hline 12 & $\mathrm{M} / 61$ & $+/+$ & 21.3 & $1.2 / 65.4$ & $1.61 / 44.8$ & 189 & $\mathrm{DN}$ \\
\hline 13 & $\mathrm{M} / 58$ & $-1-$ & 31.9 & $0.9 / 92.1$ & $1.46 / 51.5$ & 22 & Normal \\
\hline 14 & $\mathrm{~F} / 83$ & $+/+$ & $23 \cdot 3$ & $0.8 / 72.9$ & $1.68 / 28.5$ & 1 & $\mathrm{HN}$ \\
\hline 15 & $\mathrm{M} / 79$ & $+/-$ & 23.8 & $1.82 / 34.6$ & $2.15 / 28.2$ & $\mathrm{O}^{\mathrm{a}}$ & Chronic pyelonephritis \\
\hline 16 & $\mathrm{M} / 75$ & $+/+$ & 29.0 & $1.03 / 70.7$ & $1.53 / 43.8$ & 1 & MGN \\
\hline 17 & $\mathrm{M} / 71$ & $+/-$ & 19.1 & $0.73 / 93.3$ & $1.75 / 37.8$ & 3 & Ciq deposit \\
\hline 18 & $\mathrm{M} / 48$ & $-1-$ & 24.2 & $0.95 / 94.3$ & $1.44 / 56.6$ & 1 & $\mathrm{DN}$ \\
\hline 19 & $\mathrm{M} / 61$ & $-1-$ & 22.7 & $1.05 / 76.2$ & $1.72 / 41.7$ & 1 & $\mathrm{DN}$ \\
\hline
\end{tabular}

All patients received radical nephrectomy.

DM, diabetes mellitus; HTN, hypertension; BMI, body mass index; Cr, creatinine; eGFR, estimated glomerular filtration rate; DN, diabetic nephropathy; HN, hypertensive nephrosclerosis; IN, ischemic nephropathy; MGN, membranous glomerulonephritis.

${ }^{\mathrm{a}}$ These patients had pre-existing chronic kidney disease.

other pathologic processes in this study. To our knowledge, our analysis is the first to include all special stains, IF, and EM studies in pathologic evaluation of non-neoplastic kidney.

IgA nephropathy is considered the most common glomerular disease worldwide [20], and had a diagnosis rate of $17.6 \%$ in our study. During the follow-up period of 3 to 36 months, all patients were asymptomatic and their renal functions were preserved. IgA nephropathy is characterized by a highly variable course ranging from a totally benign condition to rapidly progressive renal failure [21]. Although our patients with silent IgA nephropathy showed preserved renal function during the study period, following such patients prospectively over many years may be important because chronic, slowly progressive renal failure develops in some patients with this disease [21]. Five cases revealed to have C1q deposit. Four of five patients diagnosed with C1q deposit showed normal renal function prior to nephrectomy, but following surgery, all progressed to CKD. One patient diagnosed with C1q deposit who had pre-existing CKD had an acceleration of CKD progression. There is a possibility of Ciq nephropathy of those five patients diagnosed with C1q deposit, which is known to have heterogeneous histologic findings, as well as diverse clinical presentations varying from asymptomatic to ESRD [22]. Membranous glomerulonephritis is the most common cause of nephrotic syndrome in adults [23]. Most patients with membranous glomerulonephritis present as nephrotic syndrome and the remainder are diagnosed following an evaluation for asymptomatic proteinuria [23]. Our patient who was diagnosed with membranous 
glomerulonephritis had normal urinalysis initially, but this patient had de novo CKD after nephrectomy. It is worth mentioning that all the patients diagnosed with glomerular abnormalities in our study had no known kidney disease at the time of nephrectomy. The detection of unknown kidney disease in non-neoplastic kidney tissues, although they are early lesions, may be of considerable importance to alert the physician for ongoing risk factors or causes of CKD, especially given that the solitary kidney is considered as a contraindication to percutaneous biopsy.

Our findings showing that the higher histologic scores of non-neoplastic portions could be an independent risk factor for CKD after nephrectomy further support the clinical significance of pathologic evaluation of non-neoplastic kidney. We used a simple semi-quantitative histological scoring system that has been validated in the previous studies to predict graft function after cardiac death kidney transplantations $[16,24,25]$. The degree of glomerulosclerosis, interstitial fibrosis, tubular atrophy, arteriolosclerosis, and arteriolar hyalinosis was graded from o to 3. Surprisingly, this study showed that optimal scores ( $\leq 3$ points) were observed in only $25.4 \%$ of patients and that $13.7 \%, 3.9 \%, 3.9 \%, 5.9 \%$, and $7.8 \%$ of total patients had global glomerulosclerosis, interstitial fibrosis, tubular atrophy, fibrous intimal thickening, and hyaline arteriolar thickening of grade 3. For each 1 point increase in histologic scores, the risk of CKD increased $23 \%$. These findings are in keeping with previous reports that have demonstrated that postoperative $\mathrm{sCr}$ levels increased significantly in patients with severe parenchymal changes. In a study of 110 tumor nephrectomy specimens, patients with $>20 \%$ of global glomerulosclerosis and advanced diffuse diabetic glomerulosclerosis showed a greater decline in $\mathrm{sCr}$ levels at 6-month follow-up period [17]. Among 156 patients who were followed-up for at least 12 months after tumor nephrectomy, postoperative $\mathrm{sCr}$ levels increased significantly in patients with severe arteriosclerosis or arteriolosclerosis, > $5 \%$ global glomerulosclerosis, or > 10\% interstitial fibrosis or tubular atrophy [18]. In this regard, we feel that recent update of the protocol for examination of tumor nephrectomy and nephroureterectomy specimens by the College of American Pathologists to require routine evaluation of non-neoplastic renal parenchyma is reasonable and appropriate.
Multiple studies have shown that radical nephrectomy, compared with partial nephrectomy, could lead to a substantial decline in renal function. In a study involving 1,151 patients who underwent radical nephrectomy or partial nephrectomy, radical nephrectomy nearly doubled the risk of adverse renal outcomes including ESRD, urgent dialysis, CKD, or rapidly progressive CKD when compared with partial nephrectomy [4]. Current recommendation from the American Urological Association [6] and the European Association of Urology [7] suggested partial nephrectomy as the standard of care for type 1a (T1a) RCC (tumor diameter $\leq 4 \mathrm{~cm}$ ) and as a viable option for Tib RCC (tumor diameter $\leq 7 \mathrm{~cm}$ ). In accordant with these findings, our study also showed higher event of adverse postoperative renal outcomes including AKI and CKD in radical nephrectomy. None of the partial nephrectomized patients had de novo CKD in the present study. Our findings showing that a lower preoperative eGFR and the presence of AKI were the independent risk factors for the development of postoperative CKD were consistent with the previous studies $[13,26]$.

Limitation of this study is a small sample size with short follow-up period. Further prospective studies with long-term follow-up should be performed to prove the beneficial effect of pathologic evaluation of non-neoplastic kidney in terms of early recognition of the patients at risk for CKD and timely institution of early measures to preserve their renal function.

In conclusion, our data clearly demonstrated that a thorough pathologic evaluation of non-neoplastic kidney provides valuable diagnostic and prognostic information. Therefore, routine pathologic examination of non-neoplastic parenchyma in resected tumor specimens using special stains, IF, and EM studies is recommended in the clinical practice.

\section{KEY MESSAGE}

1. The incidence of kidney cancer has been dramatically increasing worldwide, and nephrectomy remains the standard of care in the treatment of upper urinary tract malignancies, which is a risk factor of chronic kidney disease.

2. As a solitary kidney is considered as a contraindication to percutaneous biopsy and the 
safety of this procedure in patients with partial nephrectomy has not been demonstrated, thorough histologic evaluation of non-neoplastic kidney enables early identification of unrecognized kidney disease and provides valuable prognostic information. Therefore, routine pathologic examination of non-neoplastic parenchyma in resected tumor specimens is recommended in the clinical practice.

\section{Conflict of interest}

No potential conflict of interest relevant to this article was reported.

\section{Acknowledgments}

This work was supported by the Soon Chun Hyang University Research Fund.

\section{REFERENCES}

1. Sun M, Bianchi M, Hansen J, et al. Chronic kidney disease after nephrectomy in patients with small renal masses: a retrospective observational analysis. Eur Urol 2012;62:696-703.

2. Jung KW, Won YJ, Kong HJ, Oh CM, Seo HG, Lee JS. Cancer statistics in Korea: incidence, mortality, survival and prevalence in 2010. Cancer Res Treat 2013;45:1-14.

3. Sun M, Thuret R, Abdollah F, et al. Age-adjusted incidence, mortality, and survival rates of stage-specific renal cell carcinoma in North America: a trend analysis. Eur Urol 2011;59:135-141.

4. Klarenbach S, Moore RB, Chapman DW, Dong J, Braam B. Adverse renal outcomes in subjects undergoing nephrectomy for renal tumors: a population-based analysis. Eur Urol 2011;59:333-339.

5. Jeon HG, Jeong IG, Lee JW, Lee SE, Lee E. Prognostic factors for chronic kidney disease after curative surgery in patients with small renal tumors. Urology 2009;74:10641068.

6. Campbell SC, Novick AC, Belldegrun A, et al. Guideline for management of the clinical T1 renal mass. J Urol 2009;182:1271-1279.

7. Ljungberg B, Cowan NC, Hanbury DC, et al. EAU guidelines on renal cell carcinoma: the 2010 update. Eur Urol
2010;58:398-406.

8. Cho A, Lee JE, Kwon GY, et al. Post-operative acute kidney injury in patients with renal cell carcinoma is a potent risk factor for new-onset chronic kidney disease after radical nephrectomy. Nephrol Dial Transplant 2011;26:34963501.

9. Levey AS, Coresh J. Chronic kidney disease. Lancet 2012;379:165-180.

10. Go AS, Chertow GM, Fan D, McCulloch CE, Hsu CY. Chronic kidney disease and the risks of death, cardiovascular events, and hospitalization. N Engl J Med 2004;351:1296-1305.

11. Coresh J, Astor BC, Greene T, Eknoyan G, Levey AS. Prevalence of chronic kidney disease and decreased kidney function in the adult US population: Third National Health and Nutrition Examination Survey. Am J Kidney Dis 2003;41:1-12.

12. Hunsicker LG. The consequences and costs of chronic kidney disease before ESRD. J Am Soc Nephrol 2004;15:1363-1364.

13. Li L, Lau WL, Rhee CM, et al. Risk of chronic kidney disease after cancer nephrectomy. Nat Rev Nephrol 2014;10:135-145.

14. Brenner BM, Rector FC. Brenner \& Rector's the Kidney. 7th ed. Philadelphia: Saunders, 2004.

15. Levey AS, Stevens LA, Schmid CH, et al. A new equation to estimate glomerular filtration rate. Ann Intern Med 2009;150:604-612.

16. Remuzzi G, Grinyo J, Ruggenenti P, et al. Early experience with dual kidney transplantation in adults using expanded donor criteria: Double Kidney Transplant Group (DKG). J Am Soc Nephrol 1999;10:2591-2598.

17. Bijol V, Mendez GP, Hurwitz S, Rennke HG, Nose V. Evaluation of the nonneoplastic pathology in tumor nephrectomy specimens: predicting the risk of progressive renal failure. Am J Surg Pathol 2006;30:575-584.

18. Salvatore SP, Cha EK, Rosoff JS, Seshan SV. Nonneoplastic renal cortical scarring at tumor nephrectomy predicts decline in kidney function. Arch Pathol Lab Med 2013;137:531-540.

19. Henriksen KJ, Meehan SM, Chang A. Non-neoplastic renal diseases are often unrecognized in adult tumor nephrectomy specimens: a review of 246 cases. Am J Surg Pathol 2007;31:1703-1708.

20. Galla JH. IgA nephropathy. Kidney Int 1995;47:377-387.

21. Donadio JV, Grande JP. IgA nephropathy. N Engl J Med 
2002;347:738-748.

22. Vizjak A, Ferluga D, Rozic M, et al. Pathology, clinical presentations, and outcomes of Ciq nephropathy. J Am Soc Nephrol 2008;19:2237-2244.

23. Rivera F, Lopez-Gomez JM, Perez-Garcia R; Spanish Registry of Glomerulonephritis. Clinicopathologic correlations of renal pathology in Spain. Kidney Int 2004;66:898904.

24. Navarro MD, Lopez-Andreu M, Rodriguez-Benot A, et al. Significance of preimplantation analysis of kidney bi- opsies from expanded criteria donors in long-term outcome. Transplantation 2011;91:432-439.

25. Snoeijs MG, Buurman WA, Christiaans MH, et al. Histological assessment of preimplantation biopsies may improve selection of kidneys from old donors after cardiac death. Am J Transplant 2008;8:1844-1851.

26. Barlow LJ, Korets R, Laudano M, Benson M, McKiernan J. Predicting renal functional outcomes after surgery for renal cortical tumours: a multifactorial analysis. BJU Int 2010;106:489-492. 